\title{
Generation of Nonclassical Photon Pairs for Scalable Quantum Communication with Atomic Ensembles
}

\author{
A. Kuzmich, W. P. Bowen, A. D. Boozer, A. Boca, C. W. Chou, L.-M. Duan, and H. J. Kimble \\ Norman Bridge Laboratory of Physics 12-33, California Institute of Technology, Pasadena, CA 91125
}

(Dated: October 31, 2018)

\begin{abstract}
Quantum information science attempts to exploit capabilities from the quantum realm to accomplish tasks that are otherwise impossible in the classical domain [1]. Although sufficient conditions have been formulated for the physical resources required to achieve quantum computation and communication [2], there is an evolving understanding of the power of quantum measurement combined with conditional evolution for accomplishing diverse tasks in quantum information science $[3,4,5]$. In this regard, a significant advance is the invention of a protocol by Duan, Lukin, Cirac, and Zoller (DLCZ) [6] for the realization of scalable long distance quantum communication and the distribution of entanglement over quantum networks. Here, we report the first enabling step in the realization of the protocol of DLCZ, namely the observation of quantum correlations for photon pairs generated in the collective emission from an atomic ensemble. The nonclassical character of the fields is evidenced by the violation of a Cauchy-Schwarz inequality, $\tilde{g}_{1,2}^{2}(\delta t) \not \leq \tilde{g}_{1,1} \tilde{g}_{2,2}$, with $\tilde{g}_{i, j}$ as normalized corre-
\end{abstract} lation functions for the two fields $(i, j)=(1,2)$. As compared to prior investigations of nonclassical correlations for photon pairs produced in atomic cascades [7] and in parametric down conversion [8], our experiment is distinct in that the correlated $(1,2)$ photons are separated by a programmable time interval $\delta t$, with $\delta t \simeq 400$ nsec in our initial experiments.

The theoretical proposal of $D L C Z[\underline{6}]$ is a probabilistic scheme based upon the entanglement of atomic ensembles via detection events of single photons in which the sources are intrinsically indistinguishable, and generates entanglement over long distances via a quantum repeater architecture [9]. The $D L C Z$ scheme, with built-in quantum memory and entanglement purification, is well within the reach of current experiments and accomplishes the same objectives as previous more complex protocols that require still unattainable capabilities [9, 10.

In our experiment, we demonstrate a basic primitive integral to the $D L C Z$ scheme. Specifically, an initial write pulse of (classical) light is employed to create a state of collective atomic excitation as heralded by photoelectric detection of a first photon 1. After a programmable delay $\delta t$, a subsequent read pulse interrogates the atomic sample, leading to the emission of a second (delayed) photon 2. The manifestly quantum (or non- classical) character of the correlations between the initial write photon 1 and the subsequent read photon 2 is verified by way of the observed violation of a Cauchy-Schwarz inequality for coincidence detection of the $(1,2)$ fields 7 ]. Explicitly, we find $\left[\tilde{g}_{1,2}^{2}(\delta t)=(5.45 \pm 0.11)\right] \not \leq\left[\tilde{g}_{1,1} \tilde{g}_{2,2}=\right.$ $(2.97 \pm 0.08)]$, where $\tilde{g}_{i, j}$ are normalized correlation functions for the fields $(i, j)$ and $\delta t=405 \mathrm{~ns}$ is the time separation between the $(1,2)$ emissions. The capabilities realized in our experiment provide an important initial step toward the implementation of the full protocol of DLCZ, which would enable the distribution and storage of entanglement among atomic ensembles distributed over a quantum network. Extensions of these capabilities could facilitate scalable long-distance quantum communication [6] and quantum state engineering [1]. For example, by employing spin-polarized samples in optical-dipole or magnetic traps [12], it should be possible to extend the interval $\delta t$ to times of several seconds.

Our experiment arises within the context of prior work on spin squeezing [13, 14], and in particular on atomic ensembles where significant progress has been made in the development of methods to exploit collective enhancement of atom-photon interactions provided by optically thick atomic samples [15, 16, 17, 18, 19, 20]. Instead of homodyne or heterodyne detection of light as used in spin-squeezing experiments [18, 19, 20], the $D L C Z$ scheme involves photon counting techniques, which present stringent requirements for broad bandwidth detection and for the suppression of stray light from the atomic ensemble.

As illustrated in Fig. 1 an optically thick sample of three-level atoms in a lambda-configuration is exploited to produce correlated photons via the following sequence. With atoms initially prepared in state $|a\rangle$ by optical pumping, a laser pulse from the write beam tuned near the $|a\rangle \rightarrow|e\rangle$ transition illuminates the sample and induces spontaneous Raman scattering to the initially empty level $|b\rangle$ via the $|e\rangle \rightarrow|b\rangle$ transition at time $t^{(1)}$. The write pulse is made sufficiently weak so that the probability to scatter one Raman photon into the preferred forward propagating mode $\psi^{(1)}(\vec{r}, t)$ is much less than unity for each pulse. Detection of a photon in the mode $\psi^{(1)}(\vec{r}, t)$ produced by the $|e\rangle \rightarrow|b\rangle$ transition results in a single excitation in the symmetrized atomic level $|b\rangle$. In the ideal case, this coherently symmetrized state is $[\underline{6}]$

$$
\left|\Phi_{1 A}\right\rangle \sim \sum_{j=1}^{N}|a\rangle_{1} \ldots|b\rangle_{j} \ldots|a\rangle_{N} .
$$


Although the initial detection of photon 1 generated by the write beam is probabilistic, the detection of photon 1 results in the conditional state $\left|\Phi_{1 A}\right\rangle$ with one collective atomic "excitation". This excitation can subsequently be converted into an excitation of the light field with high probability "on demand" with a specified emission direction and a programmable pulse shape [6, 10, 21, 22]. In order to achieve the conversion from atoms to field, a laser pulse from the read beam tuned near the $|b\rangle \rightarrow|e\rangle$ transition illuminates the atomic sample, thereby affecting the transfer $|b\rangle \rightarrow|a\rangle$ for the sample with the accompanying emission of a second Raman photon 2 on the $|e\rangle \rightarrow|a\rangle$ transition. For an optically thick atomic sample, photon 2 is emitted with high probability into a specified mode $\psi^{(2)}(\vec{r}, t)$ offset in time by $t^{(2)}=t^{(1)}+\delta t$. The spatial and temporal structure of the modes $\psi^{(1,2)}(\vec{r}, t)$ are set by the geometry of the atomic sample and by the shape and timing of the write and read beams [21]. In our experiment, the modes of the (write, read) beams are spatially mode matched, with measured visibility greater than $95 \%$ for the case of equal frequency and polarization. The time delay $\delta t$ is limited in principle only by the coherence time between the levels $|a\rangle$ and $|b\rangle$, which is long in practice.

The atomic sample for our experiment is provided by Cesium atoms in a magneto-optical trap (MOT) 12], where the $\mathrm{Cs}$ hyperfine manifolds $\left\{\mid 6 S_{1 / 2}, F=\right.$ $\left.4\rangle,\left|6 S_{1 / 2}, F=3\right\rangle,\left|6 P_{3 / 2}, F^{\prime}=4\right\rangle\right\}$ correspond to the levels $\{|a\rangle,|b\rangle,|e\rangle\}$, respectively. As illustrated by the timing diagram in Fig. 1 the MOT is chopped from $O N$ to $O F F$ with $\Delta t=4 \mu \mathrm{s}$. In each cycle there is a "dark" period of duration $1 \mu$ s when all light responsible for trapping and cooling is gated $O F F$, with less than $0.1 \%$ of atoms measured to remain in the $F=3$ level at this stage. The $j^{\text {th }}$ trial of the protocol for single photon generation is initiated by a write pulse which is resonant with the $6 S_{1 / 2}, F=4 \rightarrow 6 P_{3 / 2}, F^{\prime}=4$ transition at frequency $\omega_{4,4}$ and that has duration $\simeq 51 \mathrm{~ns}$ (FWHM). A critical parameter for the experiment is the resonant optical thickness $\gamma_{4,4}$ of the atomic sample 21]. We measure $\gamma_{4,4} \simeq 4-5$ for cw excitation, corresponding to an attenuation of intensity $\exp \left(-\gamma_{4,4}\right)$ in propagation through the MOT.

The write pulse generates forward-scattered (antiStokes) Raman light around frequency $\omega_{3,4}$ from the $F^{\prime}=4$ excited level to the $F=3$ ground level $(|e\rangle \rightarrow|b\rangle)$ that is directed onto a single-photon detector D1. After a variable delay $\delta t$, the read pulse illuminates the sample, with this pulse tuned to the $6 S_{1 / 2}, F=3 \rightarrow$ $6 P_{3 / 2}, F^{\prime}=4$ transition at frequency $\omega_{3,4}$ with duration $\simeq 34 \mathrm{~ns}$ (FWHM). Raman (Stokes) light generated by the read pulse around frequency $\omega_{4,4}$ from $F^{\prime}=4$ to $F=4$ $(|e\rangle \rightarrow|a\rangle)$ is directed onto a second single-photon detector D2.

By interchanging the frequencies for optical pumping of the filter cells described in Fig. 11 the (write, read) beams can be detected at $(D 1, D 2)$ in place of the $(1,2)$ fields. An example of the resulting pulse profiles accumu- lated over many trials $\{j\}$ is presented in Fig. 2] where the origin in time is set to coincide with the approximate center of the write pulse, with the read pulse following after a delay $\simeq 415 \mathrm{~ns}$ determined by external control logic.

With the filter cells set to transmit the $(1,2)$ photons to the (D1, D2) detectors, respectively, we record histograms of the numbers $\left(n_{1}(t), n_{2}(t)\right)$ of photoelectric events versus time, which are also displayed in Fig. 2 For the data presented here, the intensity of the write pulse is kept low ( $\sim 10^{3}$ photons per pulse), resulting in a time lag for the onset of the $n_{1}(t)$ counts in Fig. 2 As discussed in the Supplemental Information, the probability $p_{w r i t e}^{(1)}$ to generate an anti-Stokes photon 1 within the solid angle of our imaging system is $p_{\text {write }}^{(1)} \simeq 10^{-2}$ per pulse.

The read pulse is about 100 times more intense than the write pulse, leading to high efficiency $\zeta_{3 \rightarrow 4} \simeq 0.6$ for the transfer of population $|b\rangle \rightarrow|a\rangle$, with $p_{\text {read }}^{(2)} \simeq$ $\zeta_{3 \rightarrow 4} p_{\text {write }}^{(1)}$ for the Stokes photon 2. Examples of the resulting detection events $n_{2}(t)$ are shown in Fig. 2 In contrast to the behavior of $n_{1}(t)$, the intense read beam generates $n_{2}(2)$ counts promptly. More extensive investigations of the timing characteristics of the emitted fields $(1,2)$ will be part of our subsequent investigations, including the relationship to electromagnetically induced transparency (EIT) [24, 25].

A virtue of the $D L C Z$ protocol is its insensitivity to a variety of loss mechanisms, including inefficiencies in transport and detection of the $(1,2)$ photons. However, in an actual experiment, various non-ideal characteristics of the atom-field interaction (as in our MOT) do lead to deterioration of correlation for the $(1,2)$ photons (e.g., imperfect filtering and/or background fluorescence as described in the caption of Fig. [2] and in the Supplemental Information). Fortunately there exists a well-defined border between the classical and quantum domains for the $(1,2)$ fields that can be operationally accessed via coincidence detection, as was first demonstrated in the pioneering work of Clauser [7].

As illustrated in Fig. 10, electronic pulses from detectors $(D 1, D 2)$ are separately gated with windows of duration $T=60 \mathrm{~ns}$ centered on times $\left(t^{(1)}, t^{(2)}\right)$ corresponding to the approximate peaks of the $\left(n_{1}(t), n_{2}(t)\right)$ pulses shown in Fig. 22 Photoelectric events that fall within the gate windows are directed to a time-interval analyzer (TIA) configured in a standard fashion for measurement of photoelectric correlations [26]. For a start event from $D 1$ within the interval $t_{j}^{(1)} \pm T / 2$ for the $j^{\text {th }}$ trial of the experiment, the TIA records the times of stop events from D2 within successive intervals $t_{k}^{(2)} \pm T / 2$. Over many repetitions of the experiment, we thereby acquire timeresolved coincidences $n_{1,2}(\tau)$ between the $(1,2)$ fields, both within the same trial $k=j$ and for subsequent trials $k=j+1, j+2 \ldots$ (i.e., a start event from trial $j$ around time $t_{j}$ and a stop event from trial $k$ around time $t_{k}$, where $t_{k}=t_{j}+(k-j) \Delta t$ for $\left.k=j, j+1, \ldots\right)$. By a $50-50 \%$ beam splitter, the field 1 can be directed to 
detectors (D1, D2), and then in turn the field 2 to (D1, D2). We thus also acquire the time-resolved coincidences $n_{1,1}(\tau)$ and $n_{2,2}(\tau)$.

Figure 3 displays an example of data accumulated in this manner for coincidences $n_{\alpha, \beta}(\tau)$ between the $(1,2)$, $(1,1)$, and $(2,2)$ beams, with successive peaks separated by the time between trials $\Delta t=4 \mu \mathrm{s}$. Note that there is an excess of coincidence counts in each of the initial peaks for joint detections from the same trial $(\tau<\Delta t)$ as compared to $n_{\alpha, \beta}(\tau)$ from different trials $(\tau>\Delta t)$. This excess is shown more clearly in the plots in the right column, which expand the time axis from the left column in Fig. 3 Here, data from successive trials $k=j+1, \ldots j+10$ have been offset to $\tau<\Delta t$ and then averaged for comparison with $n_{\alpha, \beta}(\tau)$ from the same trial $j$ by introducing the quantity $m_{\alpha, \beta}(\tau)=\frac{1}{10} \sum_{k=j+1}^{j+10} n_{\alpha, \beta}(\tau+(k-j) \Delta t)$. As discussed in the Supplemental Information, statistical independence for trials with $k \neq j$ is enforced by the experimental protocol of reapplying the MOT and repuming beams after each trial.

From the data in Fig. 3 we determine the total number of coincidences $N_{\alpha, \beta}=\sum_{\left\{\tau_{i}\right\}} n_{\alpha, \beta}\left(\tau_{i}\right)$ with $(\alpha, \beta)=$ $(1,2)$ obtained by summing over time bins $\left\{\tau_{i}\right\}$ for detection within the same trial $j$, and $M_{\alpha, \beta}=\sum_{\left\{\tau_{k}\right\}} m_{\alpha, \beta}\left(\tau_{k}\right)$ obtained from start and stop events from different trials $(j \neq k)$. Fields for which the Glauber-Sudarshan phasespace function is well-behaved (i.e., classical fields) are constrained by a Cauchy-Schwarz inequality for the various coincidence counts [Supplemental Information and Ref. [26]], namely

$$
\left[\tilde{g}_{1,2}(\delta t)\right]^{2} \leq \tilde{g}_{1,1} \tilde{g}_{2,2},
$$

where $\tilde{g}_{1,1} \equiv \frac{N_{1,1}}{M_{1,1}}, \tilde{g}_{2,2} \equiv \frac{N_{2,2}}{M_{2,2}}, \tilde{g}_{1,2}(\delta t) \equiv \frac{N_{1,2}}{M_{1,2}}$.

For the data displayed in Fig. 3. we find $\tilde{g}_{1,1}=$ $(1.739 \pm 0.020)$ and $\tilde{g}_{2,2}=(1.710 \pm 0.015)$, in correspondence to the expectation that the $(1,2)$ fields should each exhibit Gaussian statistics with $\tilde{g}_{1,1}=\tilde{g}_{2,2}=2$ for the protocol of $D L C Z$ in the ideal case, but here degraded by diverse sources of background counts (see Supplemental Information). By contrast, for the cross-correlations of the $(1,2)$ fields, we record $\tilde{g}_{1,2}(\delta t)=(2.335 \pm 0.014)$, with $\delta t=405 \mathrm{~ns}$. Hence the inequality of Eq. Q for classical fields is strongly violated, namely $\left[\tilde{g}_{1,2}^{2}(\delta t)=\right.$ $5.45 \pm 0.11] \not \leq\left[\tilde{g}_{1,1} \tilde{g}_{2,2}=2.97 \pm 0.08\right]$, where all errors indicate the statistical uncertainties. This violation of the Cauchy-Schwarz inequality clearly demonstrates the nonclassical character of the correlations between photons $(1,2)$ generated by the (write, read) beams. Moreover, as discussed in more detail in the Supplemental Information, the measured coincidence rates in Fig. 3 explicitly document the cooperative nature of the emission process. Overall, we estimate that the probability $p_{c}^{(q)}$ for coincidence of the $(1,2)$ photons due to collective atomic excitation as described by the state $\left|\Phi_{1 A}\right\rangle$ is roughly $p_{c}^{(q)} \simeq 10^{-4}$ for each trial $j$, referenced to the output of the MOT.

The temporal extent of the photon wave packet $\psi(\vec{r}, t)$ for the $(1,2)$ photons is also of some interest. To investigate this issue, we have carried out the experiment with expanded gate windows of duration $T=140 \mathrm{~ns}$ that then encompass the entire domains over which counts $n_{1}(t)$ and $n_{2}(t)$ are observed in Fig. 2] In this case, we record $\tilde{g}_{1,1}=(1.72 \pm 0.04), \tilde{g}_{2,2}=(1.52 \pm 0.05)$, and $\tilde{g}_{1,2}(\delta t)=(2.45 \pm 0.10)$, now with $\delta t$ set to be $320 \mathrm{~ns}$. The classical inequality of Eq. 0 is once again not satisfied; $\left[\tilde{g}_{1,2}^{2}(\delta t)=6.00 \pm 0.50\right] \not \leq\left[\tilde{g}_{1,1} \tilde{g}_{2,2}=2.61 \pm 0.11\right]$. These results with $T=140$ ns also confirm that deadtime effects do not play a significant role in the current experiment.

As described in the Supplemental Information, the violation of the Cauchy-Schwarz inequality of Eq. 2] in the ideal case can be much larger than we have observed, namely $\left[\tilde{g}_{1,2}(\delta t)\right]^{2} /\left[\tilde{g}_{1,1} \tilde{g}_{2,2}\right] \simeq[(1+p) /(2 p)]^{2} \gg$ 1 , where $p \ll 1$ is the excitation probability. In our experiment, the size of the violation of the inequality was limited mostly by uncorrelated fluorescence from individual atoms in the atomic sample. This contribution will be made smaller in future experiments by moving to off-resonant excitation, which necessitates higher optical density. There is also a significant limitation due the presence of the leakage light from the read pulse. This classical pulse is only $9 \mathrm{GHz}$ away from the single-photon field 2 of interest, and is filtered by a factor exceeding $10^{-9}$. To achieve even stronger violation of the inequality, we must further improve the filtering capability.

Our observations of nonclassical correlations between the $(1,2)$ photons represent the first important step in the realization of the protocol of $D L C Z[\underline{6}]$ for scalable quantum communication with atomic ensembles, although it is not yet sufficient for realization of the full protocol. Beyond the nonclassical correlations, our experiment also demonstrates successful filtering of the various fields and collective enhancement by the atomic ensemble, all of which are critical for realization of the full quantum repeater protocol. More generally, the capabilities that we have demonstrated should help to enable other advances in the field of quantum information, including implementation of quantum memory [22, 27] and fully controllable single-photon sources [28], which, combined together, help to pave the avenue for realization of universal quantum computation [4].
[1] Quantum computation, Preskill J. P., Lecture Notes for Physics 219/Computer Science 219, http://www.theory.caltech.edu/people/preskill/ph229/

[2] DiVincenzo, D. P., The physical implementation of quan- 
tum computation. Fortschr. Phys., 48 (2000) 771-783.

[3] Bose S., Knight P. L., Plenio M. B., and Vedral V., Proposal for teleportation of an atomic state via cavity decay. Phys. Rev. Lett. 83, 5158-5161 (1999).

[4] Knill, E., Laflamme, R. \& Milburn G. J. A scheme for efficient quantum computation with linear optics. Nature 409 , 46-52 (2001).

[5] Raussendorf, R., and Briegel, H. J., A one-way quantum computer. Phys. Rev. Lett. 86, 5188-5191 (2001).

[6] Duan, L.-M., Lukin, M., Cirac, J. I., and Zoller, P., Longdistance quantum communication with atomic ensembles and linear optics. Nature 414, 413 (2001).

[7] Clauser, J. F., Experimental distinction between the quantum and classical field-theoretic predictions for the photoelectric effect. Phys. Rev. D 9, 853-860 (1974).

[8] Mandel, L., Quantum effects in one-photon and twophoton interference. Rev. Mod. Phys. 71 (2): S274-S282 (1999).

[9] Briegel, H.-J., Duer, W., Cirac, J. I. \& Zoller, P. Quantum repeaters: The role of imperfect local operations in quantum communication. Phys. Rev. Lett. 81, 5932-5935 (1999).

[10] Enk, S. J., Cirac, J. I., and Zoller, P., Photonic channels for quantum communication. Science 279, 205-208 (1998).

[11] Duan L.-M., Entangling many atomic ensembles with laser manipulation. Phys. Rev. Lett. 88, 170402-170405 (2002).

[12] Laser Cooling and Trapping, Metcalf H. J. and van der Straten P. (Springer-Verlag, 1999).

[13] Kitagawa M., and Ueda M., Nonlinear-interferometric generation of number-phase correlated fermion states. Phys. Rev. Lett. 67, 1852-1854 (1991).

[14] Wineland D. J., Bollinger J. J., Itano W. M., and Moore F. L., Spin squeezing and reduced quantum noise in spectroscopy, Phys. Rev. A 46, R6797-R6800 (1992).

[15] Kuzmich A., Mølmer K., and Polzik E. S., Spin Squeezing in an Ensemble of Atoms Illuminated with Squeezed Light, Phys. Rev. Lett. 79, 4782 (1997).

[16] Kuzmich A., Bigelow N. P., and Mandel L., Atomic quantum non-demolition measurements and squeezing, Europhys. Lett. A 42, 481-486 (1998).

[17] Mølmer K., Twin-correlations in atoms, Eur. Phys. J. D 5, 301-305 (1999).

[18] Hald J., Sørensen J. L., Schori C., and Polzik E. S., Spin squeezed atoms: a macroscopic entangled ensemble created by light, Phys. Rev. Lett. 83, 1319-1320 (1999).

[19] Kuzmich A., Mandel L., and Bigelow N. P., Generation of spin squeezing via continuous quantum non-demolition measurements, Phys. Rev. Lett. 85, 1594-1597 (2000).

[20] Julsgaard B., Kozhekin A., and Polzik E. S., Experimental long-lived entanglement of two macroscopic objects, Nature 413, 400-403 (2001).

[21] Duan L.-M., Cirac J. I., and Zoller P., Three-dimensional theory for interaction between atomic ensembles and freespace light. Phys. Rev. A 66, 023818 (2002).

[22] Fleischhauer, M., Lukin, M. D. Dark-state polaritons in electromagnetically induced transparency. Phys. Rev. Lett. 84, 5094-5097 (2000).

[23] Alexandrov E. B., Balabas M. V., Pasgalev A. S., Verkhovskii A. K., and Yakobson N. N., Doubleresonance atomic magnetometers: from gas discharge to laser pumping, Laser Physics 6, 244-251 (1996).

[24] Harris S. E., Hau L. V., Nonlinear optics at low light levels, Phys. Rev. Lett. 82, 4611-4614 (1999).

[25] Zibrov A. S., Matsko A. B., Kocharovskaya O., Rostovtsev Y.V., Welch G. R., Scully M. O., Transporting and time reversing light via atomic coherence, Phys. Rev. Lett. 88, 103601-1-103601-4 (2002).

[26] Optical Coherence and Quantum Optics, Mandel L. and Wolf E. (Cambridge University Press, 1995).

[27] Schori C., Julsgaard B., Sørensen J. L., and Polzik E. S., Recording Quantum Properties of Light in a Long-Lived Atomic Spin State: Towards Quantum Memory,Phys. Rev. Lett. 89, 057903-1-057903-4 (2002).

[28] Pelton M. et al., Efficient source of single photons: A single quantum dot in a micropost microcavity. Phys. Rev. Lett. 89, 233602 (2002).

Acknowledgements HJK gratefully acknowledges interactions with M. D. Lukin regarding various aspects of the experiment and its interpretation. This work was supported by the National Science Foundation, by the Caltech MURI Center for Quantum Networks under ARO Grant No. DAAD19-00-1-0374, and by the Office of Naval Research.

Competing interests statement The authors declare that they have no competing financial interests.

Correspondence and requests for materials should be addressed to H.J.K. (e-mail: hjkimble@caltech.edu). 


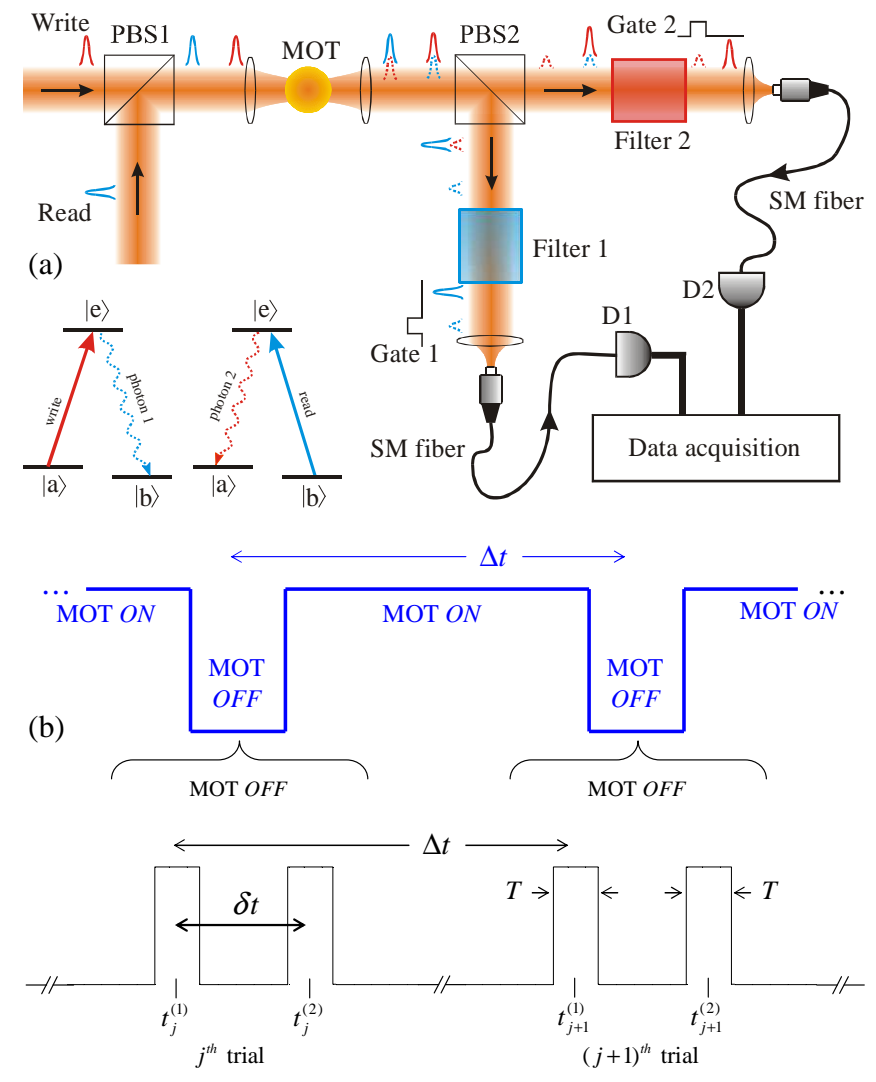

FIG. 1: A simplified schematic of the experiment is presented, with (a) providing a diagram of the apparatus and (b) giving the timing sequence for data acquisition. Further details are as follows. (a) Write and read pulses propagate sequentially into a cloud of cold Cs atoms (MOT), generating pairs of correlated output photons $(1,2)$, with controlled separation $\delta t$. Fields with frequency near that of the $|a\rangle \leftrightarrow|e\rangle(|b\rangle \leftrightarrow|e\rangle)$ transition are colored red (blue) here and in the subsequent two figures. The write and read pulses have orthogonal polarizations, are combined into a single input at PBS1 (PBS polarizing beam splitter), and are then focussed into the Cs MOT with a waist of approximately $30 \mu \mathrm{m}$. The output fields are split by PBS2, which also serves as the first stage of filtering the (write, read) beams from the $(1,2)$ fields. For example, field 2 is transmitted by PBS2 to be subsequently registered by detector $D 2$, while the read pulse itself is reflected by $90^{\circ}$ at PBS2 and then blocked by an acousto-optical modulator that serves as Gate 1. Further filtering is achieved by passing each of the outputs from PBS2 through separate frequency filters each of which consists of a glass cell of Cs vapor optically pumped to place atoms into either $6 S_{1 / 2}, F=3$ or $F=4$ [23]. The small residual reflected (transmitted) light of the write (read) pulse from PBS2 at frequency $\omega_{4,4}\left(\omega_{3,4}\right)$ passes through a filter cell with atoms in the $F=4(3)$ level. It is thereby strongly attenuated $\left(>10^{6}\right)$, while the accompanying Raman-scattered light as photons 1(2) at frequency $\omega_{3,4}\left(\omega_{4,4}\right)$ is transmitted with high efficiency $(\simeq 80 \%)$. Transmission efficiencies from the MOT to detectors $(D 1, D 2)$ are both about $30 \%$ for light with the spatial shape of the write and read beams and of the correct polarization. (D1, D2) have overall quantum efficiencies of approximately $50 \%$ (photon in to TTL pulse out). (b) Gating windows for the joint detection of photons $(1,2)$ are centered at times $\left(t_{j}^{(1)}, t_{j}^{(2)}\right)$ for the $j^{\text {th }}$ trial of the experiment during intervals when the MOT is $O F F$.

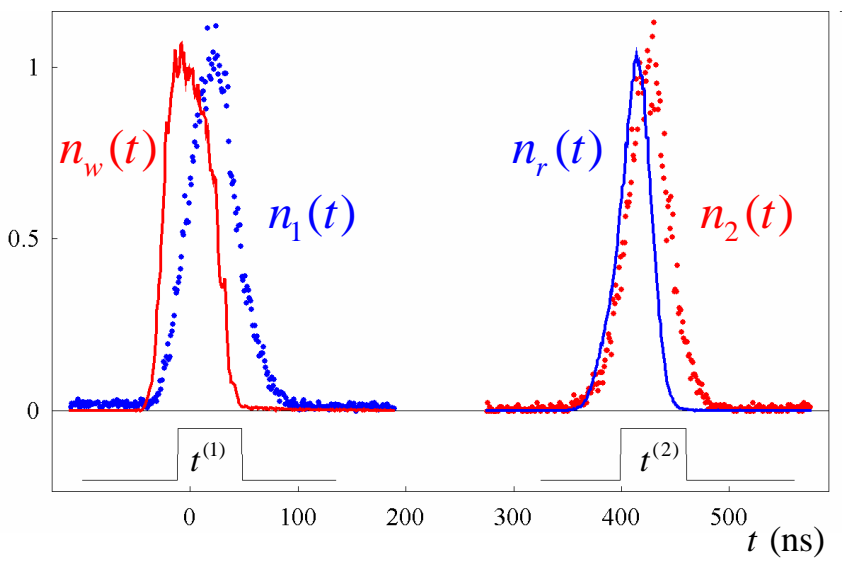

FIG. 2: Normalized singles counts $n_{i}(t)$ are shown for the write, read, and $(1,2)$ fields. The pulses around $t=0$ are from detector $D 1$ for the write beam $n_{w}(t)$ (solid trace) and for photon $1, n_{1}(t)$ (points). The pulses around $t=410 \mathrm{~ns}$ are from detector D2 for the read beam $n_{r}(t)$ (solid trace) and for photon $2, n_{2}(t)$ (points). Note that in addition to the symmetrized excitation, each write pulse also transfers several hundred atoms into the $F=3$ level due to spontaneous emission from its near-resonant character. However, atoms transferred into $F=3$ via spontaneous decay are spatially uncorrelated, so that their contribution to the signal from the read channel is strongly suppressed (by roughly the fractional solid angle collected, $\delta \Omega / 4 \pi \simeq 4 \times 10^{-5}$ ) as compared to the signal from single-atom excitations of the form $\left|\Phi_{1 A}\right\rangle[6]$. 

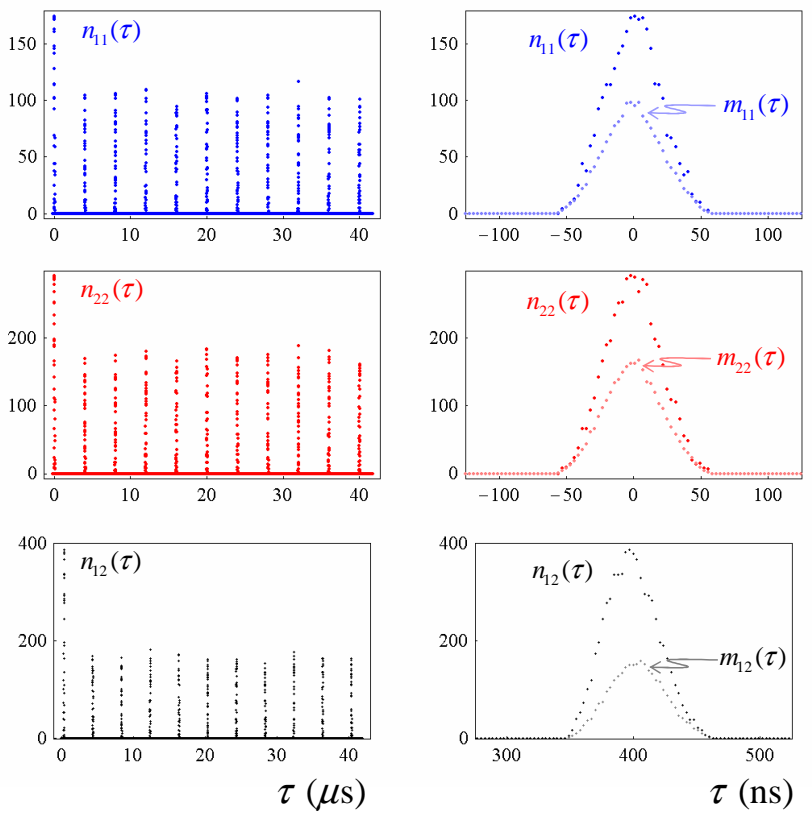

FIG. 3: Time-resolved coincidences $n_{\alpha, \beta}(\tau)$ between the $(1,1),(2,2)$, and $(1,2)$ fields are displayed versus time delay $\tau$. Left column $-n_{\alpha, \beta}(\tau)$ is shown over 11 successive repetitions of the experiment. Right column - The time axis is expanded to a total duration of 250ns with $\tau=0$ set to the center of the gating window $\left(t^{(1)}, t^{(2)}, t^{(1)}\right)$ for $\left(n_{11}, n_{22}, n_{12}\right)$, respectively. The larger peak $n_{\alpha, \beta}(\tau)$ corresponds to detection pairs from the same trial $j$, while the smaller peak $m_{\alpha, \beta}(\tau)$ is for pairs from different trails as defined in the text. Typical acquisition parameters are as follows. Detectors $(D 1, D 2)$ have average count rates of about (400/s, 250/s), respectively, while background counts with no MOT present are about 100/s. Counts due to the MOT itself (with write and read beams blocked) are less than $(10 / \mathrm{s}, 20 / \mathrm{s})$ for $(D 1$, D2). Dark counts with the inputs to the fibers blocked are less than $5 / \mathrm{s}$. All these numbers are for the gated-output mode of data acquisition as in Fig. 1 with $T=60 \mathrm{~ns}$. 